3

4 Elsa Wagner-Rousset ${ }^{1 *}$, Szabolcs Fekete ${ }^{2}$, Laura Morel-Chevillet ${ }^{1}$, Olivier Colas ${ }^{1}$, Nathalie 5 Corvaïa ${ }^{1}$, Davy Guillarme ${ }^{2}$, Alain Beck*1.

$6 \quad{ }^{*}$ Corresponding authors:

\section{Development of a fast workflow to screen the charge variants of therapeutic antibodies.}

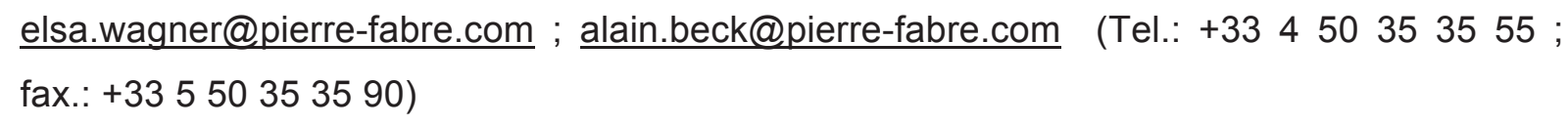

${ }^{1}$ Centre d'Immunologie Pierre Fabre, 5 Avenue Napoléon III, 74160 Saint-Julien-en-Genevois, France.

${ }^{2}$ School of Pharmaceutical Sciences, University of Geneva, University of Lausanne, Boulevard d'Yvoy 20, 1211 Geneva 4, Switzerland.

\section{Abstract}

Chemical or enzymatic modifications of therapeutic monoclonal antibodies (mAbs) having high risk towards safety and efficacy are defined as critical quality attributes (CQAs). During therapeutic mAbs process development, a variety of analytical techniques have to be used for the thorough characterization and quantitative monitoring of CQAs. This paper describes the development of a rapid analytical platform to assess and rank charge variants of mAbs. The workflow is first based on a cation exchange chromatography (CEX) comparative analysis of intact IgGs versus $F(a b)$ '2 and Fc sub-domains generated by IdeS digestion. This analytical procedure was validated with FDA and EMA approved mAbs. Then, functional assays and peptide mapping can be performed in a second instance. This approach can be used during the early stage of drug research and development to screen lead molecules and select optimized candidates (best clone, best formulation) which could be "easily" developed (OptimAbs).

\section{Keywords}

Cation exchange chromatography; charge variants; developability; IdeS; trastuzumab. 
Many post-translational modifications as well as physical and chemical degradations of mAbs have been described in the past twenty years [1]. The number and type of modifications might affect surface charge of the monoclonal antibody (mAb), either by directly changing the number of charged residues or by altering the surface charge distribution (Fig. 1). Numerous variants are commonly observed when analyzing mAbs with charged-based separation techniques such as iso-electric focusing (IEF) gel electrophoresis, capillary isoelectric focusing (cIEF) gel electrophoresis, imaged capillary isoelectric focusing (iclEF), cation exchange chromatography (CEX) and anion exchange chromatography (AEX). These variants are generally referred as acidic or basic species, when compared to the main isoform. Acidic species are variants with low apparent isoelectric point ( $\mathrm{pl}$ ), and basic species are variants with high apparent pl, when antibodies are analyzed using IEF-based methods. When using chromatographic-based methods, acidic and basic species are defined based on their retention times relative to the main peak. Acidic species are, for example, the variants that elute before the main peak by CEX or after the main peak by AEX. Charge variants include $\mathrm{N}$-terminal cyclization of glutamine or glutamic acid residues at both heavy and light chains. Clipping of the C-terminal side of the heavy chain is also commonly observed: clipping of the C-terminal lysine (or when previously removed by molecular engineering), clipping of the C-terminal glycine simultaneously with amidation of the neighboring prolines. Less frequently, lysine $-\mathrm{NH}_{2}$ moieties aldimination, acetonation and glycation also impact the mAb surface charge distribution [2]. Sialic acids described in $\mathrm{CHO}$, $\mathrm{SP} 2 / 0$ or NS0 produced IgGs carry negative charges. Finally, deamidation of asparagine residues may occur, preferentially at neutral or basic $\mathrm{pH}$. Deamidation takes place via formation of an intermediate succinimide, which is unstable and hydrolyzes into a mixture of two acidic isomers Asp and IsoAsp. Deamidation may also occur at acidic $\mathrm{pH}$ by direct hydrolysis of the side chain amide yielding only Asp. To a lesser extent, isoAsp may originate from Asp isomerization [3]. The importance of those charge modifications in terms of safety, immunogenicity and efficacy have been evaluated and ranked by Liu et al. [4]. Modifications are considered as highly critical when they are only observed in recombinant lgG, and less risky when they are common to endogenous IgG. The ranking of importance according to Liu et al. has been illustrated in Fig. 1. For example, N-terminal pyroglutamic residues are variants naturally occurring in IgG, not considered as critical quality attributes. On the other hand, deamidation in complementarity determining regions (CDRs) occur after inappropriate $\mathrm{pH}$ buffer storage 
charge variants of a recombinant humanized IgG1 were isolated and compared, showing no difference in FcRn binding affinity, pharmacokinetic properties in rats and in vitro potency [5]. Charge related heterogeneity of mAbs is mainly characterized with IEF [6] or IEX [7]. CEX is adapted for molecules with basic isoelectric points, such as mAbs [7]. In CEX, the positive net charge of $\mathrm{mAbs}$ under $\mathrm{pH}$ conditions of the experiment would interact with negatively charged stationary phase. Elution is then performed either by $\mathrm{pH}$ or salt gradient (or combination of both). The separation of the variants depends on the net-surface charge, charge distribution and conformation of the protein [8]. In CEX, acidic variants such as deamidated, sialylated, glycated compounds elute before the main peak, while basic ones (species with C-terminal lysine, C-terminal proline amidation, uncyclized $\mathrm{N}$-terminal glutamine) elute after the main peak [9].

Whereas charge modifications of constant domains are usually common to each lgG subclass, modifications affecting variable domains require a special focus and need to be studied regardless of their potential impact on mAb binding and activity. Papain has been used so far to fragment recombinant mAbs into Fab and Fc fragments that could be chromatographically separated. Moorhouse et al. [10] developed an assay for determining the product purity of a papain-digested mAb, looking at the charge heterogeneity of each fragment. Vlasak et al. reported an extensive work based on papain digestion, followed by CEX separation and peptide mapping of isolated variants to aid identification of the degradation products of a mAb [11]. Moreover, they demonstrated a LC CDR1-isoAsp variant which was cleaved by papain in an adjacent CDR2 loop, exhibiting greater flexibility than the native form (i.e. asparagine containing form). The idea to investigate critical quality attributes of mAbs by CEX following a highly specific cleavage in the hinge area is thus attractive. It became possible with the introduction of IdeS enzyme, which rapidly generates homogenous pool of $F(a b ') 2$ and Fc fragments without over-digestion or further degradation [12]. The benefits of IdeS for mAb and ADC characterization by LC-MS at middle level has been recently shown[13,14].

The purpose of our work is to demonstrate, using a panel of commercial and in-house therapeutic mAbs, the potency of IdeS digested mAbs prior to CEX separation and MS investigation for in depth and relevant charge variants identification. Assuming that Fc charge variants have limited impact on mAb activity $[15,16]$, we therefore focused during the study on CDRs containing $\mathrm{F}(\mathrm{ab})$ '2.

Robust linear $\mathrm{pH}$ gradient methods have been developed by providers for analyzing antibody charge heterogeneity[17-19]. A clear advantage of those approaches over conventional salt gradient elution methods is their broad applicability for a wide range of IgG, without the need for individual optimization [7,20]. Nevertheless, in one of our recent study, $\mathrm{pH}$ and salt gradient modes were comparatively evaluated for a panel of $10 \mathrm{mAbs}$ with pl ranking from 
6.7 to 9.1, showing that both can be considered as "multi-product charge sensitive separation methods". Whereas selectivity were quite comparable, charge variants were better resolved in the salt gradient mode and peak capacities were generally improved with this approach $[21,22]$. In the present study, both approaches were applied to the study of seven IdeS digested mAbs, and only the chromatograms resulting in the best variants separation were shown.

\section{Materials \& methods}

Water was produced from a Milli-Q Water Purification System ${ }^{\mathrm{TM}}$ from Millipore. All chemicals and reagents were of analytical grade and purchased from Sigma-Aldrich or VWR International. IdeS was purchased from Genovis (FabRICATOR Kit, Genovis). For IdeS sample digestion, mAbs were diluted in water to a final concentration of $5 \mathrm{mg} / \mathrm{mL}$ and digested with 1 unit IdeS per $\mu \mathrm{g}, 30 \mathrm{~min}$ at $37^{\circ} \mathrm{C} .1 \mathrm{M}$ 2-(N-morpholino)ethanesulfonic acid (MES) solution (BioReagent), $1 \mathrm{M}$ sodium hydroxide $(\mathrm{NaOH})$ solution were purchased from Sigma-Aldrich. $\mathrm{CEX} \mathrm{pH}$ gradient buffer $\mathrm{A}(\mathrm{pH}=5.6)$ and $\mathrm{CEX} \mathrm{pH}$ gradient buffer $\mathrm{B}(\mathrm{pH}=$ 10.2) were purchased from Thermo Fisher Scientific AG.

\subsection{Cation exchange chromatography}

All the experiments were performed using an HPLC system consisting in two 510 pumps, a 717 auto-injector and a 490 UV detector (Waters). Thermo MAbPac SCX-10, 4 x 250 mm, 10 $\mu \mathrm{m}$, strong cation exchange column was purchased from Thermo Fisher Scientific. The flow rate was set at $1 \mathrm{~mL} / \mathrm{min}$. The $\mathrm{mAb}$ samples (either digested or not with IdeS) were diluted in water to a final concentration of $1 \mathrm{mg} / \mathrm{mL}$. A volume of $20 \mu \mathrm{L}$ was injected using low volume insert vials. A linear salt gradient from 0 to $20 \%$ B in 20 minutes was applied for analysis of adalimumab and rituximab. For the salt gradient CEX separation, the mobile phase "A" consisted of $10 \mathrm{mM}$ MES in water, while the mobile phase "B" was $10 \mathrm{mM}$ MES in water containing $1 \mathrm{M} \mathrm{NaCl}$. The $\mathrm{pH}$ of both mobile phases was adjusted by adding $1 \mathrm{M} \mathrm{NaOH}$ solution to attain a $\mathrm{pH}$ of 5.7. For the $\mathrm{pH}$ gradient CEX separation, the mobile phase "A" was a ten times diluted CEX $\mathrm{pH}$ gradient buffer $\mathrm{A}(\mathrm{pH}=5.6)$, while the mobile phase "B" was a ten times diluted $\mathrm{CEX} \mathrm{pH}$ gradient buffer $\mathrm{B}(\mathrm{pH}=10.2)$. $\mathrm{A} \mathrm{pH}$ gradient from $0 \%$ to $100 \% \mathrm{~B}$ was applied in 30 minutes. The chromatographic signal was recorded at a wavelength of 280 $\mathrm{nm}$.

\subsection{Mass spectrometry}


Peptide mapping was performed by LC-MS ${ }^{\mathrm{E}}$ after trypsin digestion of collected fractions on a

141 Synapt G2Si equipped with an Acquity UPLC H-class system (Waters, Milford, MA, USA).

142 Digestion protocol and MS method were described in Xie et al. [23].

143

144

145

146

147

148

149

150

151

152

153

154

155

156

157

158

159

160

161

162

163

164

165

166

167

168

169

170

171

172

173

174

175

176

\section{Results and discussion}

In this study, seven therapeutic mAbs were selected, as they are representative of the most common IgGs on the market (table 1). Six of them were humanized IgG1K, one was chimeric IgG1K. Their pl ranged from 8.7 to 9.2 , an alkaline range well adapted to the salt gradient mode CEX described in Fekete et al. [21,22]. Adalimumab, bevacizumab, rituximab, trastuzumab and dalotuzumab sequences are available in the IMGT database. Two in-house products (mAbPF1 and mAbPF2) were also included. In Fig. 2, the chromatograms resulting from either salt or $\mathrm{pH}$ gradient mode CEX are presented, for the intact $\mathrm{mAb}$, and IdeS digested $m A b$. The separation of both $F(a b ') 2$ and $F c$ parts was good enough to consider charge variants related to each fragment. Whatever is the separation method, the Fc fragment (which has lower $\mathrm{pl}$ ) elute first at a retention time of around 8 to 10 minutes, while the $F(a b ') 2$ fragment could elute at a different retention time, depending on its sequence. In the experimental conditions, based on the use of non-denaturating chromatographic elution buffer, we assume that the Fc fragment was detected as a non-covalently bound dimer. Despite the lack of hinge region after IdeS digestion, weak interactions between both Fc/2 subunits were shown to be sufficient to maintain dimerization in ammonium acetate buffer, when analyzed in off-line CZE-native MS [24]. Thus, this consideration impact the way how the data were interpreted, especially when considering possible heterodimers, due to partial C-terminal lysine clipping or partial proline amidation.

\subsection{Fc charge variants}

Sequence alignment of $F c$ fragment of the seven mAbs is presented in Fig. 3. A few differences can be noticed, especially on the amino acids at position $120 \& 122$ (see the numbering of figure 3), which may vary upon the mAb allotype. Adalimumab and rituximab are G1m 17,1 regarding their heavy chains, which means they have D356 \& L358 instead of E356 \& M358 (HC numbering) [25,26]. This allotype feature might not induce any change in the charge profile of Fc fragment. Moreover, five out of the seven mAbs still have C-terminal lysine giving raise to partial K-clipping, as commonly described [5,27]. Therefore, basic charge variants were observed and annotated as "K1" and "K2" in reference to the main peak "K0". On the other hand, mAbPF1 and PF2 have both engineered C-termini sides without lysine at their heavy chains. Nevertheless, C-terminal proline amidation, catalyzed by peptidylglycine alpha-hydroxylating monooxygenase (PAM) in the $\mathrm{CHO}$ cells expressing mAbs, might explain basic variants observed in the charge profiles (-PCONH2)[28,29]. 
Finally, asparagine deamidation of the Fc domain may be considered. As depicted in Fig. 3, Fc domain of IgG1 contains ten asparagine residues, which were monitored by peptide

179

180

\subsection{Adalimumab}

Adalimumab charge variants were extensively characterized by CIEF and CEX [31]. When treated by carboxypeptidase B, three peaks collapsed into lower pl (CIEF) or shorter retention time (CEX) zones, demonstrating that charge variants were due to the numbers of $C$-terminal heavy chain lysine. Moreover, CEX analysis of the papain digestion of the anti-TNF mAb indicated that the isoforms belong to the Fc part. In the same way, IdeS digested adalimumab (Fig. 2) displays one $F\left(a b^{\prime}\right) 2$ and one Fc fragment, carrying the charge heterogeneity due to C-terminal lysine (K1 \& K2). Both fragments present small acidic peaks $(3 \%)$. Since the product was registered in 2002 , adalimumab manufacturing period includes changes in the production process and scale that required addressing consistency of its quality attribute. Representative CEX chromatograms showed that charge microheterogeneity of adalimumab drug substance, as measured by the proportion of lysine variants was maintained [32].

\subsection{Bevacizumab}

For bevacizumab, the proportion of basic variants, due to C-terminal lysine, was very low (5\% for Fc-K1 \& K2 after IdeS digestion, see Fig. 2) resulting from a near complete clipping. This difference in charge heterogeneity was due to varying degree of proteolysis by carboxypeptidases (Cps). C-terminal lysine variants were found to be sensitive to process conditions, especially components in culture medium such as lysine and arginine amounts, whereas $\mathrm{pH}$ and $\mathrm{Cps}$ levels do not impact the variant proportion [33]. The residual proportion of C-terminal lysine directly impact complement-dependent cytotoxicity (CDC). Indeed, C1q 
binding and $C D C$ requires $\lg$ hexamerization at the cell surface, which is optimal when

214 lysine are fully removed by Cps [34].

\section{4. $\quad \underline{\text { Rituximab }}$}

217 In the case of rituximab, IdeS digestion allows simplification of the mAb basic charge profile. 218 The Fc fragment carries $10 \%$ of the modifications, due to residual C-terminal lysine, while the $219 \mathrm{~F}(\mathrm{ab}) 2$ fragment carries charge heterogeneity due to the N-termini of LC and HC (12\%). 220 Cyclization occurs through the rearrangement of the parent reactive GIn residues. Since 221 pyroglutamate $(\mathrm{pE})$ loss a primary amine during the conversion, rituximab becomes more 222 acidic. Incomplete cyclization results in heterogeneity which is observed as multiple peaks. 223 Basic variants of rituximab were shown to significantly decrease along the life of this 224 biopharmaceutical product. Indeed, for batches with expiry dates in 2010 or later (here 2014), the relative amounts of basic variants were reduced from $\sim 30-50 \%$ to $\sim 10 \%$, indicating an alteration of the product related to a change in manufacturing process [35]. Both $\mathrm{N}$-terminal cyclisation and $\mathrm{C}$-terminal lysine clipping are phenomena naturally occurring in IgGs, which do not impact safety, PK and PD, and therefore are not considered as critical quality attributes (CQA). Such variations illustrate the range of micro-variations that can be accepted by health authorities [36].

\subsection{Dalotuzumab}

The CEX charge variants profile of dalotuzumab has been previously elucidated. As shown in Fig. 2, the chromatogram of intact mAb still gives comparable qualitative results as the one published. The two basic variants observed on intact $m A b$ and $F c$ fragment were interpreted as C-terminal lysine variants on heavy chains. CEX and IEF analysis combined with carboxypeptidase B confirmed this hypothesis [37]. Within an extensive work, including papain digestion, CEX fractionation plus Edman degradation and Isoquant, Vlasak et al. characterized the acidic variants. They were mostly due to deamidation of Asn33 located in the first LC complementary determining region (CDR1) leading to Asp and isoAsp variants [11]. This Asn, followed by a glycine residue, was thus clearly identified as a deamidation hot spot. Although the two variants Asp and isoAsp have identical net charge, they were chromatographically separated, suggesting some level of structural differences [11]. It is known that isoAsp residues from Asn deamidation tend to gradually isomerize to Asp. According to $F\left(a b^{\prime}\right) 2$ fragment from IdeS digestion (and not Fab, as reported in Vlasak et al.), the acidic profile does reflect heterogeneity from Asp and isoAsp occurring on possibly one or two arms. Without any further investigation, it is therefore neither possible to discriminate between Asp and isoAsp, nor to determinate the proportion of each of them in the acidic $F(a b ') 2$ fraction. 


\subsection{Trastuzumab}

252 Trastuzumab charge variants have been extensively analyzed and a number of modifications 253 in the CDRs were reported as critical quality attributes (CQAs) following functional evaluation $254[1,38]$. Indeed, trastuzumab sequence contains three hot spots in the CDRs: -Asn ${ }^{30}$-Thr- (LC) 255 ; -Asn ${ }^{55}$-Gly- $(\mathrm{HC})$; -Asp ${ }^{102}$-Gly-(HC). Seven forms of rhuMAb HER2 could be resolved by 256 CEX using a combination of analytical techniques [39]. Four acidic variants contain, either at

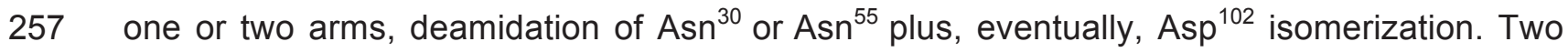
258 basic variants are due to single isomerization or succinimide from $A_{s p}{ }^{102}$. In a clear manner, 259 those seven peaks are well resolved in the $F\left(a b^{\prime}\right) 2$ from CEX profile on Fig. 2 and could be 260 identified by homology and consistency with published data. Potency assays measured the 261 ability of the antibody to prevent the proliferation of a cell line derived from a human 262 adenocarcinoma that over-expresses her2/neu gene [39]. The form with one deamidated 263 light chain was shown to have a slightly lower potency, whereas the form with isoAsp ${ }^{102}$ in one heavy chain CDR was greatly affected. Those results were consistent with previous studies demonstrating that trastuzumab antiproliferative activity requires a bivalent antibody 266 [40].

3.7. $\underline{\text { mAbPF1 }}$

This in-house recombinant antibody reveals quite homogeneous profiles. Nevertheless, basic charge variants from untreated mAb were collected, and then analyzed by LC-MS after IdeS digestion and reduction (data not shown). Light chain and Fd fragments displayed masses in agreement with the ones calculated from sequences. However, Fc/2 displayed a loss of -58 Da when compared to the expected one, which fits with a C-terminal glycine deletion and C274 terminal alpha-amidated proline, and is consistent with the shift in retention time observed by 275 CEX. C-terminal amidation has been reported for the first time by Johnson et al. [41] and 276 further described as resulting from effect of copper concentration in cell culture media of 277 IgG1 mAbs [28]. This modification affecting the Fc fragment of mAbPF1 is clearly illustrated 278 in Fig. 2, within two basic variants (12\%) either related to one or both arms of the mAb. 279 Moreover, N-terminal loss of ammonia leading to pyroglutamic acid residue formation may result in the few acidic variants of $F\left(a b^{\prime}\right) 2$. This was confirmed by direct Edman degradation of the whole $\mathrm{mAb}$ after digestion with pyroglutamate aminopeptidase $[37,42,43]$. 


\section{8. $\mathrm{mAbPF2}$}

MAbPF2 CEX profile is heterogeneous for several reasons. IdeS digested charge variants profile were particularly helpful for interpretation. Indeed, basic variants observed on the intact $\mathrm{mAb}$ profile are, from one major part, due to C-terminal proline amidation following glycine deletion (as above described), but also to residual glutamine residues that were not fully converted to pyroglutamate. Both modifications affect $F c$ or $F(a b$ ')2 CEX profiles, respectively and were confirmed thanks to complementary experiments (data not shown). Acidic fractions might have been enhanced by the formulation buffer $\mathrm{pH}$ (Tris- $\mathrm{HCl} 100 \mathrm{mM}$, $\mathrm{pH}$ 7.5) and were correlated to deamidation. Deamidation of the Fc part has been previously discussed in section 3.1, and our attention was therefore focused on the acidic fraction of the $\mathrm{F}\left(a b^{\prime}\right) 2$ fragment. The chromatographic separation was good enough to collect the corresponding products. We have thus performed tryptic peptide mapping by LC-MS ${ }^{E}$. Especially, T11, a CDR peptide of the heavy chain containing an asparagine at position 108, has retained our attention. As depicted in Fig. 4, its extracted ion chromatogram results in three separated peaks which were interpreted as $N 108, D^{108}$, iso $D^{108}$ and confirmed by fragmentation spectra (focus on the area of interest on A \& B, Fig. 4). Deamidated forms which represent $28 \%$ could not been discriminated by MS since they are isobaric. It is, from our knowledge, the first time that deamidation occurring on a -NY- sequence is described.

\section{Conclusions}

Thanks to the described workflow, it is possible to discriminate critical and less critical charge variants, according to the CEX charge variants profile of IdeS digested mAbs. This is an important feature since screening of hot spots is an unavoidable part of a developability platform. As mentioned by Jarasch et al. [44], despite the recent progress to predict degradation-prone asparagine and aspartate residues, the functional impact of such degradation can currently only be experimentally assessed. As an example, mAbPF2 highlights a non-described deamidation hot spot, -NY-, which was evidenced after short stress study and investigation of the Fab'2 fragment. As reported in this study, CEX appears as a fast and reliable technique for the characterization of charge variants. However, one of its limitations is the difficulty to identify the observed acidic and basic variants. To solve this issue, it is possible to collect some fractions and then reanalyze the samples with LC-MS, but this procedure is time-consuming. Recently, Stoll et al. have reported the possibility to directly identify CEX peaks using an on-line CEX x RPLC-MS platform [45-48], which could be particularly useful to speed up the identification of charge variants.

\section{Acknowledgments}


319 Davy Guillarme wish to thank the Swiss National Science Foundation for support through a 320 fellowship to Szabolcs Fekete (31003A 159494).

321 The authors thank Claire Catry for its valuable assistance to format the document. 
[1] A. Beck, E. Wagner-Rousset, D. Ayoub, A. Van Dorsselaer, S. Sanglier-Cianferani, Characterization of therapeutic antibodies and related products, Anal. Chem. 85 (2013) 715-736.

[2] J.F. Valliere-Douglass, L. Connell-Crowley, R. Jensen, P.D. Schnier, E. Trilisky, M. Leith, B.D. Follstad, J. Kerr, N. Lewis, S. Vunnum, M.J. Treuheit, A. Balland, A. Wallace, Photochemical degradation of citrate buffers leads to covalent acetonation of recombinant protein therapeutics, Protein Sci. 19 (2010) 2152-2163.

[3] W. Zhang, M.J. Czupryn, Analysis of isoaspartate in a recombinant monoclonal antibody and its charge isoforms, J. Pharm. Biomed. Anal. 30 (2003) 1479-1490.

[4] H. Liu, G. Ponniah, H.M. Zhang, C. Nowak, A. Neill, N. Gonzalez-Lopez, R. Patel, G. Cheng, A.Z. Kita, B. Andrien, In vitro and in vivo modifications of recombinant and human IgG antibodies, mAbs 6 (2014) 1145-1154.

[5] L.A. Khawli, S. Goswami, R. Hutchinson, Z.W. Kwong, J. Yang, X. Wang, Z. Yao, A. Sreedhara, T. Cano, D. Tesar, I. Nijem, D.E. Allison, P.Y. Wong, Y.H. Kao, C. Quan, A. Joshi, R.J. Harris, P. Motchnik, Charge variants in IgG1: Isolation, characterization, in vitro binding properties and pharmacokinetics in rats, MAbs 2 (2010) 613-624.

[6] R. Gahoual, A. Beck, E. Leize-Wagner, Y.N. Francois, Cutting-edge capillary electrophoresis characterization of monoclonal antibodies and related products, $\mathrm{J}$. Chromatogr. B 1032 (2016) 61-78.

[7] S. Fekete, A. Beck, J. Fekete, D. Guillarme, Method development for the separation of mAbs charge variants in cation exchange chromatography, part I: Salt gradient approach, J. Pharm. Biomed. Anal. 102 (2015) 33-44.

[8] J. Vlasak, R. Ionescu, Heterogeneity of Monoclonal Antibodies relvealed by chargesensitive methods, Curr. Pharm. Biotechnol. 9 (2008) 468-481.

[9] O.O. Dada, N. Jaya, J. Valliere-Douglass, O. Salas-Solano, Characterization of acidic and basic variants of IgG1 therapeutic monoclonal antibodies based on non-denaturing IEF fractionation, Electrophoresis (2015) doi: 10.1002/elps.201500219.

[10] K.G. Moorhouse, W. Nashabeh, J. Deveney, N.S. Bjork, M.G. Mulkerrin, T. Ryskamp, Validation of an HPLC method for the analysis of the charge heterogeneity of the recombinant monoclonal antibody IDEC-C2B8 after papain digestion, J. Pharm. Biomed. Anal. 16 (1997) 593-603.

[11] J. Vlasak, M.C. Bussat, S. Wang, E. Wagner-Rousset, M. Schaefer, C. KlinguerHamour, M. Kirchmeier, N. Corvaia, R. Ionescu, A. Beck, Identification and characterization of asparagine deamidation in the light chain CDR1 of a humanized IgG1 antibody, Anal. Biochem. 392 (2009) 145-154.

[12] J. Sjogren, F. Olsson, A. Beck, Rapid and improved characterization of therapeutic antibodies and antibody related products using IdeS digestion and subunit analysis, Analyst 141 (2016) 3114-3125. 
[13] E. Wagner-Rousset, M.C. Janin-Bussat, O. Colas, M. Excoffier, D. Ayoub, J.F. Haeuw, I. Rilatt, M. Perez, N. Corvaia, A. Beck, Antibody-drug conjugate model fast characterization by LC-MS following IdeS proteolytic digestion, mAbs 6 (2014) 273-285.

[14] Y. Leblanc, M. Romanin, N. Bihoreau, G. Chevreux, LC-MS analysis of polyclonal IgGs using IdeS enzymatic proteolysis for oxidation monitoring, J. Chromatogr. B, 961 (2014) $1-4$.

[15] S. Sinha, L. Zhang, S. Duan, T.D. Williams, J. Vlasak, R. lonescu, E.M. Topp, Effect of protein structure on deamidation rate in the Fc fragment of an IgG1 monoclonal antibody, Protein Sci. 18 (2009) 1573-1584.

[16] Y.T. Zhang, J. Hu, A.L. Pace, R. Wong, Y.J. Wang, Y.S. Kao, Characterization of asparagin 330 deamidation in an Fc-fragment of IgG1 using cation exchange chromatography and peptide mapping, J. Chromatogr. B 965 (2014) 65-71.

[17] J.C. Rea, G.T. Moreno, Y. Lou, D. Farnan, Validation of a pH gradient-based ionexchange chromatography method for high-resolution monoclonal antibody charge variant separations, J. Pharm. Biomed. Anal. 54 (2011) 317-323.

[18] N. Lingg, M. Berndtsson, B. Hintersteiner, M. Schuster, M. Bardor, A. Jungbauer, Highly linear $\mathrm{pH}$ gradients for analyzing monoclonal antibody charge heterogeneity in the alkaline range: Validation of the method parameters, J. Chromatogr. A., 1373 (2014) 124-130.

[19] N. Lingg, E. Tan, B. Hintersteiner, M. Bardor, A. Jungbauer, Highly linear pH gradients for analyzing monoclonal antibody charge heterogeneity in the alkaline range, J. Chromatogr. A., 1319 (2013) 65-71.

[20] D. Farnan, G.T. Moreno, Multiproduct high-resolution monoclonal antibody charge variant separations by $\mathrm{pH}$ gradient ion-exchange chromatography, Anal. Chem. 81 (2009) 8846-8857.

[21] S. Fekete, A. Beck, J. Fekete, D. Guillarme, Method development for the separation of monoclonal antibody charge variants in cation exchange chromatography, Part II: $\mathrm{pH}$ gradient approach, J. Pharm. Biomed. Anal. 102 (2015) 282-289.

[22] S. Fekete, A. Beck, D. Guillarme, Characterization of cation exchanger stationary phases applied for the separations of therapeutic monoclonal antibodies, J. Pharm. Biomed. Anal. 111 (2015) 169-176.

[23] H. Xie, A. Chakraborty, J. Ahn, Y.Q. Yu, D.P. Dakshinamoorthy, M. Gilar, W. Chen, S.J. Skilton, J.R. Mazzeo, Rapid comparison of a candidate biosimilar to an innovator monoclonal antibody with advanced liquid chromatography and mass spectrometry technologies, mAbs 2 (2010) 379-394.

[24] Y.N. Francois, M. Biacchi, N. Said, C. Renard, A. Beck, R. Gahoual, E. Leize-Wagner, Characterization of cetuximab Fc/2 dimers by off-line CZE-MS, Anal. Chim. Acta. 908 (2016) 168-176.

[25] R. Jefferis, M.P. Lefranc, Human immunoglobulin allotypes: possible implications for immunogenicity, mAbs 1 (2009) 332-338.

[26] D. Ternant, C. Arnoult, M. Pugniere, C. Dhommee, D. Drocourt, E. Perouzel, C. Passot, N. Baroukh, D. Mulleman, G. Tiraby, H. Watier, G. Paintaud, V. Gouilleux-Gruart, IgG1 
Allotypes Influence the Pharmacokinetics of Therapeutic Monoclonal Antibodies through FcRn Binding, J. Immunol. 196 (2016) 607-613.

[27] G. Walsh, R. Jefferis, Post-translational modifications in the context of therapeutic proteins, Nature Biotechnol. 24 (2006) 1241-1252.

[28] T. Kaschak, D. Boyd, F. Lu, G. Derfus, B. Kluck, B. Nogal, C. Emery, C. Summers, K. Zheng, R. Bayer, A. Amanullah, B. Yan, Characterization of the basic charge variants of a human IgG1: effect of copper concetration in cell culture media, mAbs 3 (2011) 577-583.

[29] M. Tsubaki, I. Terashima, K. Kamata, A. Koga, C-terminal modification of monoclonal antibody drugs: amidated species as a general product-related substance. Int. J. Biol. Macromol. 52 (2013) 139-147.

[30] Y.D. Liu, J.Z. van Enk, G.C. Flynn, Human antibody Fc deamidation in vivo, Biologicals 37 (2009) 313-322.

[31] L.C. Santora, I.S. Krull, K. Grant, Characterization of recombinant human monoclonal tissue necrosis factor-alpha antibody using cation-exchange HPLC and capillary isoelectric focusing, Anal. Biochem. 275 (1999) 98-108.

[32] P.W. Tebbey, A. Varga, M. Naill, J. Clewell, J. Venema, Consistency of quality attributes for the glycosylated monoclonal antibody Humira(R) (adalimumab), mAbs 7 (2015) 805-811.

[33] F. Zhang, J.H. Yang, K. Dai, Y. Chen, Q.R. Li, F.M. Gao, R.J. Zeng, Characterization of microbial compositions in a thermophilic chemostat of mixed culture fermentation, Appl. Microbiol. Biotechnol. 100 (2016) 1511-1521.

[34] E.T. van den Bremer, F.J. Beurskens, M. Voorhorst, P.J. Engelberts, R.N. de Jong, B.G. van der Boom, E.M. Cook, M.A. Lindorfer, R.P. Taylor, P.H. van Berkel, P.W. Parren, Human IgG is produced in a pro-form that requires clipping of C-terminal lysines for maximal complement activation, mAbs 7 (2015) 672-680.

[35] M. Schiestl, T. Stangler, C. Torella, T. Cepeljnik, H. Toll, R. Grau, Acceptable changes in quality attributes of glycosylated biopharmaceuticals, Nat. Biotechnol. 29 (2011) 310312.

[36] A. Beck, H. Diemer, D. Ayoub, F. Debaene, E. Wagner-Rousset, C. Carapito, A. Van Dorsselaer, S. Sanglier-Cianferani, Analytical characterization of biosimilar antibodies and Fc-fusion proteins, Trends Anal. Chem. 48 (2013) 81-95.

[37] A. Beck, M.C. Bussat, N. Zorn, V. Robillard, C. Klinguer-Hamour, S. Chenu, L. Goetsch, N. Corvaia, A. Van Dorsselaer, J.F. Haeuw, Characterization by liquid chromatography combined with mass spectrometry of monoclonal anti-IGF-1 receptor antibodies produced in CHO and NSO cells, J. Chromatogr. B 819 (2005) 203-218.

[38] M. Haberger, K. Bomans, K. Diepold, M. Hook, J. Gassner, T. Schlothauer, A. Zwick, C. Spick, J.F. Kepert, B. Hienz, M. Wiedmann, H. Beck, P. Metzger, M. Molhoj, C. Knoblich, U. Grauschopf, D. Reusch, P. Bulau, Assessment of chemical modifications of sites in the CDRs of recombinant antibodies: Susceptibility vs. functionality of critical quality attributes, mAbs 6 (2014) 327-339. 
[39] R.J. Harris, B. Kabakoff, F.D. Macchi, F.J. Shen, M. Kwong, J.D. Andya, S.J. Shire, N. Bjork, K. Totpal, A.B. Chen, Identification of multiple sources of charge heterogeneity in a recombinant antibody, J. Chromatogr. B 752 (2001) 233-245.

[40] R.F. Kelley, M.P. O'Connell, P. Carter, L. Presta, C. Eigenbrot, M. Covarrubias, B. Snedecor, J.H. Bourell, D. Vetterlein, Antigen binding thermodynamics and antiproliferative effects of chimeric and humanized anti-p185HER2 antibody Fab fragments, Biochemistry 31 (1992) 5434-5441.

[41] K.A. Johnson, K. Paisley-Flango, B.S. Tangarone, T.J. Porter, J.C. Rouse, Cation exchange-HPLC and mass spectrometry reveal C-terminal amidation of an IgG1 heavy chain, Anal. Biochem. 360 (2007) 75-83.

[42] A. Beck, M.C. Bussat, C. Klinguer-Hamour, L. Goetsch, J.P. Aubry, T. Champion, E. Julien, J.F. Haeuw, J.Y. Bonnefoy, N. Corvaia, Stability and CTL activity of N-terminal glutamic acid containing peptides, J. Pept. Res. 57 (2001) 528-538.

[43] A. Beck, C. Klinguer-Hamour, M.C. Bussat, T. Champion, J.F. Haeuw, L. Goetsch, T. Wurch, M. Sugawara, A. Milon, A. Van Dorsselaer, T. Nguyen, N. Corvaia, Peptides as tools and drugs for immunotherapies, J. Pept. Sci. 13 (2007) 588-602.

[44] A. Jarasch, H. Koll, J.T. Regula, M. Bader, A. Papadimitriou, H. Kettenberger, Developability assessment during the selection of novel therapeutic antibodies, J. Pharm. Sci., 104 (2015) 1885-1898.

[45] M. Alvarez, G. Tremintin, J. Wang, M. Eng, Y.H. Kao, J. Jeong, V.T. Ling, O.V. Borisov, On-line characterization of monoclonal antibody variants by liquid chromatographymass spectrometry operating in a two-dimensional format, Anal. Biochem. 419 (2011) 17-25.

[46] D.R. Stoll, D.C. Harmes, J. Danforth, E. Wagner, D. Guillarme, S. Fekete, A. Beck, Direct identification of rituximab main isoforms and subunit analysis by online selective comprehensive two-dimensional liquid chromatography-mass spectrometry, Anal. Chem. 87 (2015) 8307-8315.

[47] D.R. Stoll, J. Danforth, K. Zhang, A. Beck, Characterization of therapeutic antibodies and related products by two-dimensional liquid chromatography coupled with UV absorbance and mass spectrometric detection, J. Chromatogr. B 1032 (2016) 51-60.

[48] M. Sorrensen, D.C. Harmes, D.R. Stoll, G.O. Gregory, S. Fekete, D. Guillarme, A. Beck, Comparison of originator and biosimilar therapeutic mAbs using comprehensive 2D-LC with TOF MS, mAbs 8 (2016) 1224-1234. 


\section{Figure 1:}

484 IgG charge micro-variants and their importance (from Liu et al. [3]). ++++ indicates the 485 highest level of importance; + indicates the lowest level of importance. Adapted from Beck et 486 al [1]. Modifications that are common to recombinant mAb and endogenous IgG molecules 487 are considered to pose a lower risk of immunogenicity in patients. On the other hand, 488 modifications that are unique to recombinant mAbs could potentially pose higher risk. The 489 focus of this review is the comparison of frequently observed modifications of recombinant 490 monoclonal antibodies to those of endogenous IgG molecules.

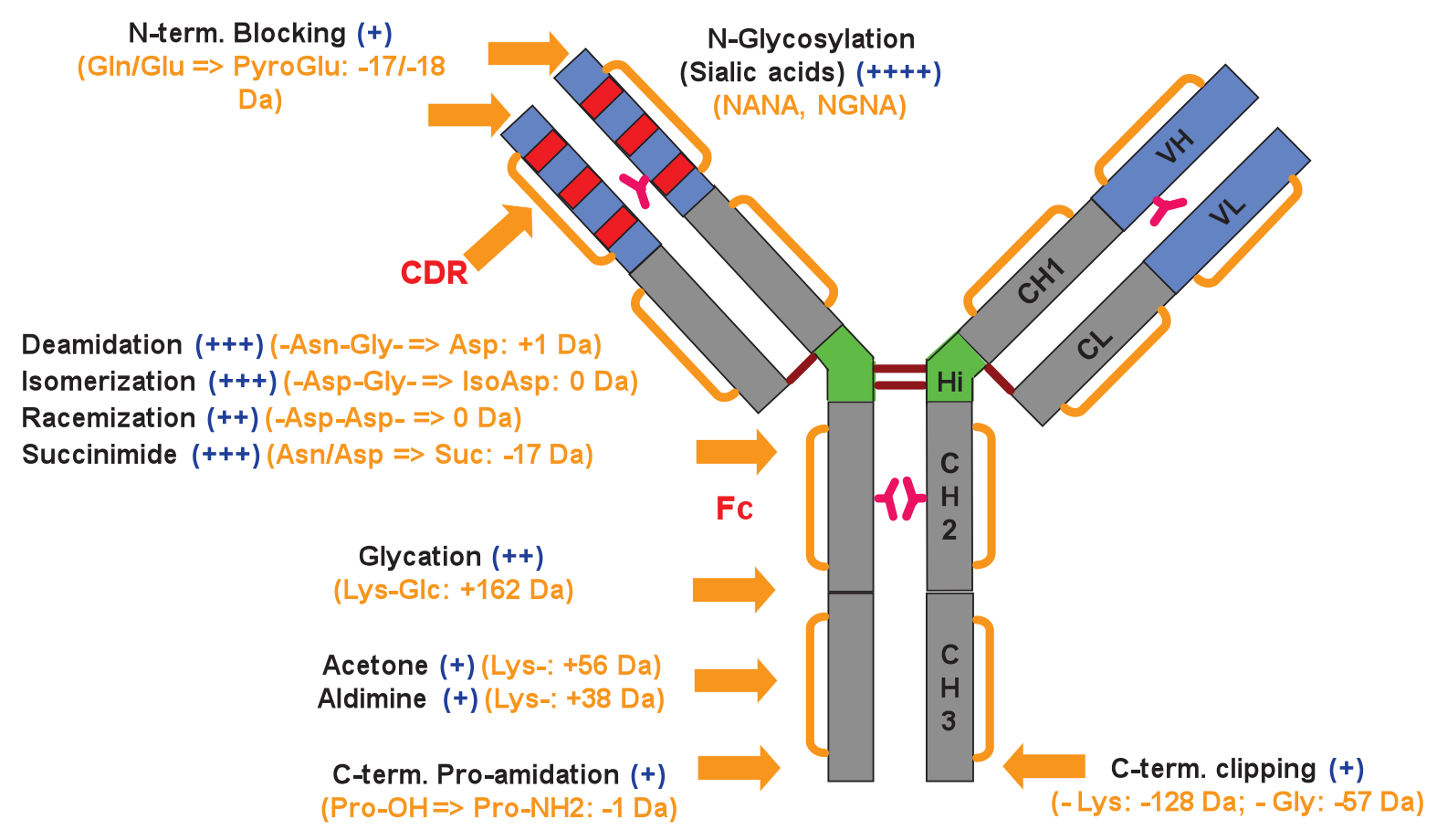


493 Figure 2:

494 CEX chromatograms of 7 therapeutic monoclonal antibodies. Each antibody was analyzed 495 either intact (chromatogram at the top) or IdeS digested (chromatogram below) within 496 identical analytical conditions (salt or $\mathrm{pH}$ gradient mode CEX, depending on the mAb). 


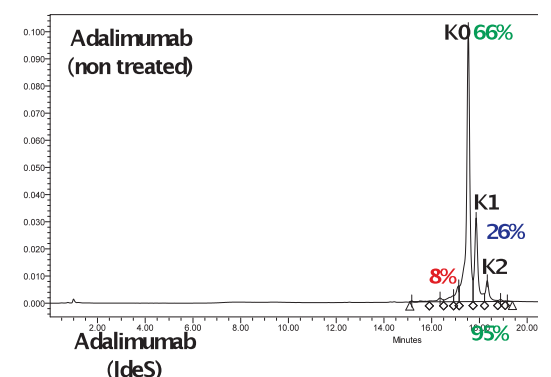

(IdeS)

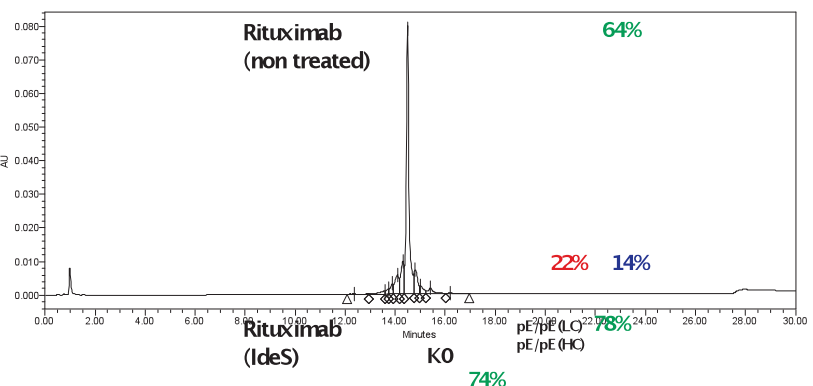

ko $73 \%$

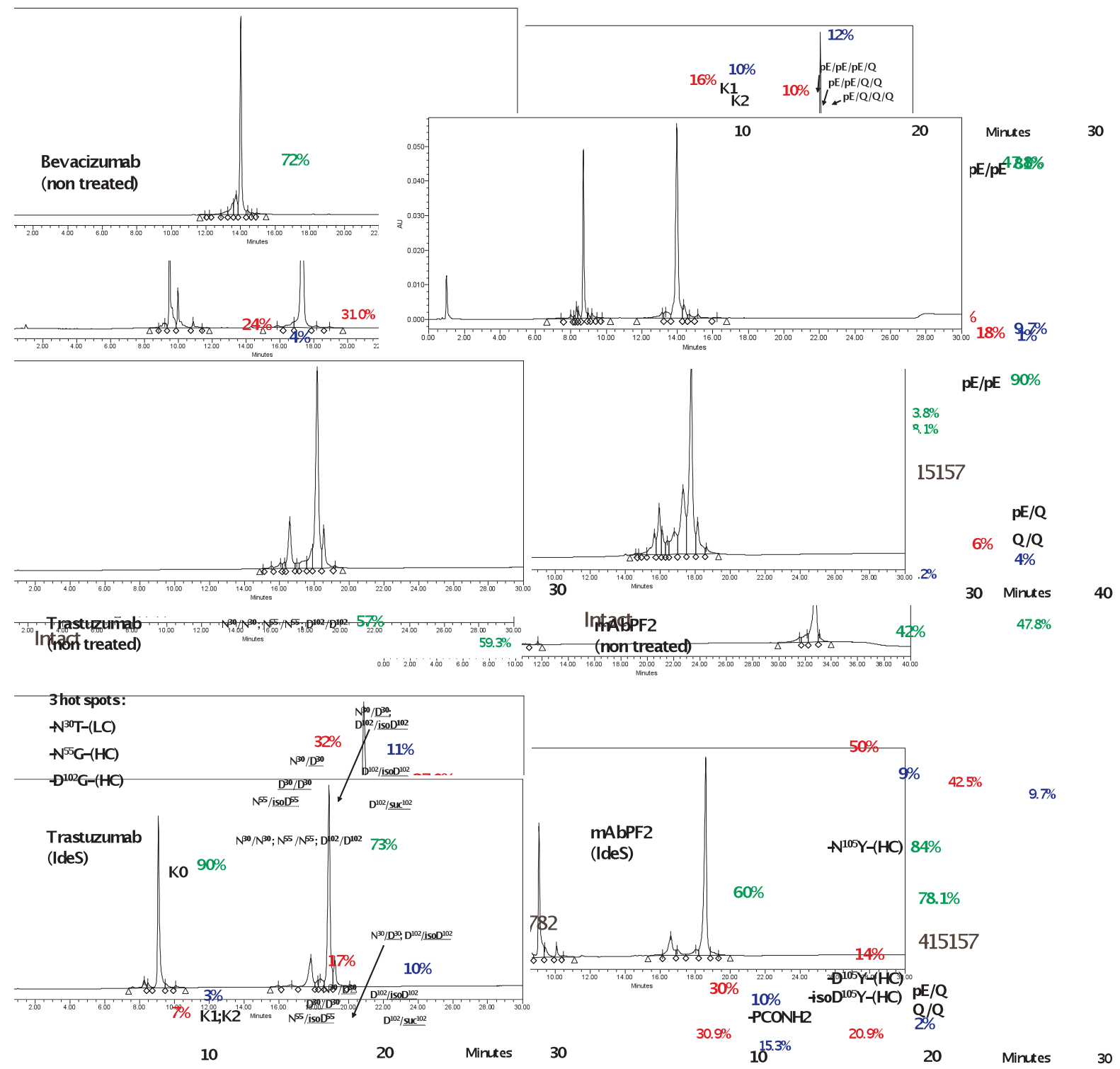


499 Figure 3:

500 Sequence alignement of Fc/2 fragments from the studied IgG1 obtained after IdeS digestion.

501 In green are highlighted differences. Asparagine are red colored.

$\begin{array}{lll} & & 1 \\ \text { Adalimumab } & (1) & \text { GPSVFLFPPKPKDTLMISRTPEVTCVVVDVSHEDPEVKFNWYVDGVEVHN } \\ \text { Bevacizumab } & (1) & \text { GPSVFLFPPKPKDTLMISRTPEVTCVVVDVSHEDPEVKFNWYVDGVEVHN } \\ \text { Rituximab } & (1) & \text { GPSVFLFPPKPKDTLMISRTPEVTCVVVDVSHEDPEVKFNWYVDGVEVHN } \\ \text { Dalotuzumab } & (1) & \text { GPSVFLFPPKPKDTLMISRTPEVTCVVVDVSHEDPEVKFNWYVDGVVHN } \\ \text { Trastuzumab } & (1) & \text { GPSVFLFPPKPKDTLMISRTPEVTCVVVDVSEDPEVKFNWYVDGVVHN } \\ \text { mAbPF1 } & (1) & \text { GPSVFLFPPKPKDTLMISRTPEVTCVVVDVSHEDPEVKFNWYVDGVEVHN } \\ \text { mAbPF2 } & (1) & \text { GPSVFLFPPKPKDTLMISRTPEVTCVVVDVSHEDPEVKFNWYVDGVEVHN } \\ & & 51\end{array}$

Adalimumab (51) AKTKPREEQYNSTYRVVSVLTVLHQDWLNGKEYKCKVSNKALPAPIEKTI Bevacizumab (51) AKTKPREEQYNSTYRVVSVLTVLHQDWLNGKEYKCKVSNKALPAPIEKTI Rituximab (51) AKTKPREEQYNSTYRVVSVLTVLHQDWLNGKEYKCKVSNKALPAPIEKTI Dalotuzumab (51) AKTKPREEOYNSTYRVVSVLTVLHODWLNGKEYKCKVSNKALPAPIEKTI Trastuzumab (51) AKTKPREEQYNSTYRVVSVLTVLHQDWLNGKEYKCKVSNKALPAPIEKTI MAbPF1 (51) AKTKPREEQYNSTYRVVSVLTVLHQDWLNGKEYKCKVSNKALPAPIEKTI MAbPF2 (51) AKTKPREEQYNSTYRVVSVLTVLHQDWLNGKEYKCKVSNKALPAPIEKTI

101

150

Adalimumab Bevacizumab Rituximab

Dalotuzumab Trastuzumab $\mathrm{mAbPF} 1$

mAbPF 2

(101) SKAKGQPREPQVYTLPPSRDELTKNQVSLTCLVKGFYPSDIAVEWESNGQ

(101) SKAKGQPREPQVYTLPPSREEMTKNQVSLTCLVKGFYPSDIAVEWESNGQ

(101) SKAKGQPREPQVYTLPPSRDELTKNQVSLTCLVKGFYPSDIAVEWESNGQ

(101) SKAKGQPREPQVYTLPPSREEMTKNQVSLTCLVKGFYPSDIAVEWESNGQ

(101) SKAKGQPREPQVYTLPPSREEMTKNQVSLTCLVKGFYPSDIAVEWESNGQ

(101) SKAKGQPREPQVYTLPPSREEMTKNQVSLTCLVKGFYPSDIAVEWESNGQ

(101) SKAKGQPREPQVYTLPPSREEMTKNQVSLTCLVKGFYPSDIAVEWESNGQ

151

200

Adalimumab Bevacizumab Rituximab

Dalotuzumab

Trastuzumab

mAbPF 1

mAbPF2

(151) PENNYKTTPPVLDSDGSFFLYSKLTVDKSRWQQGNVFSCSVMHEALHNHY

(151) PENNYKTTPPVLDSDGSFFLYSKLTVDKSRWQQGNVF SCSVMHEALHNHY

(151) PENNYKTTPPVLDSDGSFFLYSKLTVDKSRWQQGNVFSCSVMHEALHNHY

(151) PENNYKTTPPVLDSDGSFFLYSKLTVDKSRWQQGNVF SCSVMHEALHNHY

(151) PENNYKTTPPVLDSDGSFFLYSKLTVDKSRWQQGNVFSCSVMHEALHNHY

(151) PENNYKTTPPVLDSDGSFFLYSKLTVDKSRWQQGNVFSCSVMHEALHNHY

(151) PENNYKTTPPVLDSDGSFFLYSKLTVDKSRWQQGNVFSCSVMHEALHNHY

Adalimumab Bevacizumab Rituximab Dalotuzumab Trastuzumab mAbPF 1 


\section{Figure 4:}

505 Mass spectrometry analysis of isolated acidic fraction of mAbPF2 from CEX put in evidence 506 deamidation in a non-expected $N$ at positon 108 in CDR3 of the heavy chain. On figure 4A, is 507 presented the extracted ion chromatogram (EIC) of the tryptic peptide $\mathrm{T} 11(\mathrm{HC})$ containing 508 the CDR3 deamidation site. The chromatogram displays 3 peaks: peaks $2 \& 3$ represent $28 \%$ 509 of the total area. MS spectra corresponding to chromatographic peaks 1, $2 \& 3$ are displayed 510 on figure 4B showing at mass shift of +1 Da for peaks 2 and 3 when compared to MS 511 spectrum of peak 1. Additional MS/MS spectra (figure $4 \mathrm{C}$ ) confirm the sequence of T11(HC) 512 peptide: non deamidated for peak 1 ; deamidated for peak 2.

513
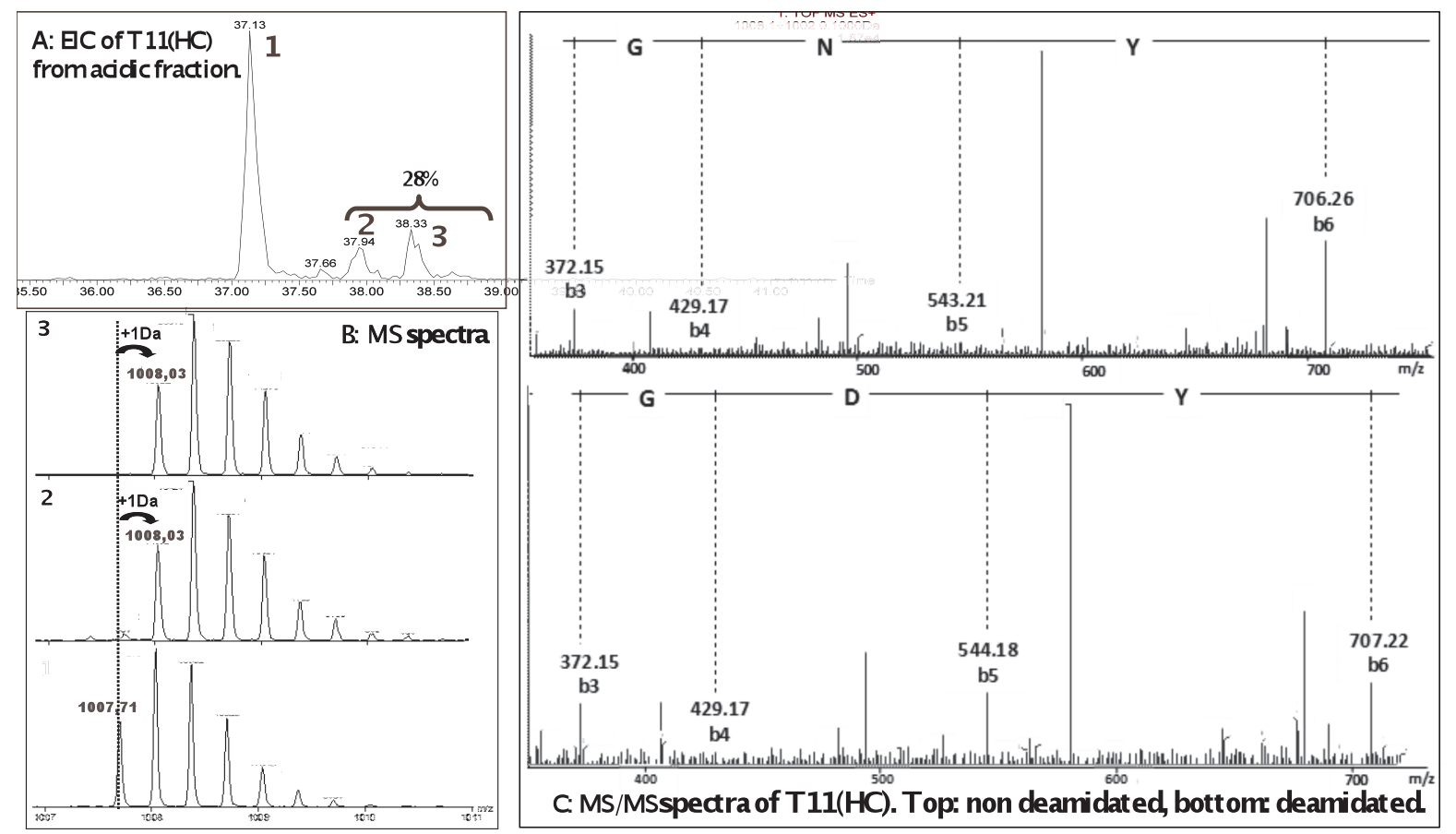
$516 \quad$ Table 1

517 Summary of mAbs described in the study and their attributes.

\begin{tabular}{|c|c|c|c|c|c|c|c|}
\hline $\begin{array}{c}\text { INN } \\
\text { (International } \\
\text { Nonproprietary Name) }\end{array}$ & $\begin{array}{l}\text { Target } \\
\text { name }\end{array}$ & Indications & Format & Company & $\begin{array}{c}\text { Proprietary } \\
\text { name }\end{array}$ & $\begin{array}{c}\text { Calculated } \\
\text { pl }\end{array}$ & $\begin{array}{c}\text { CEX } \\
\text { method }\end{array}$ \\
\hline adalimumab & TNF & $\begin{array}{l}\text { rheumatoid arthritis, } \\
\text { crohn disease and } \\
\text { others }\end{array}$ & hlgG1k & AbbVie Inc. & HUMIRA & 8.7 & salt gradien \\
\hline bevacizumab & VEGF & $\begin{array}{l}\text { collorectal cancer } \\
\text { metastatic and others }\end{array}$ & hzlgG1k & $\begin{array}{l}\text { Roche, F. Hoffmann- } \\
\text { La Roche } \\
\text { LtD./Genetech Inc. }\end{array}$ & AVASTIN & 8.5 & $\mathrm{pH}$ gradient \\
\hline rituximab & CD20 & $\begin{array}{l}\text { non-hodgkin's } \\
\text { lymphoma }\end{array}$ & clgG1k & $\begin{array}{l}\text { Biogen, Inc, Roche, } \\
\text { F. Hoffmann-La } \\
\text { Roche } \\
\text { LtD./Genetech Inc. }\end{array}$ & $\begin{array}{l}\text { RITUXAN } \\
\text { (US, Canada, } \\
\text { Japan); } \\
\text { MABTHERA } \\
\text { (EU) }\end{array}$ & 9.1 & salt gradien \\
\hline dalotuzumab & IGF1R & Solid tumors & hzlgG1k & Merck / Pierre Fabre & $\begin{array}{c}\mathrm{n} / \mathrm{a} \\
\text { (Phase II / III) }\end{array}$ & 9.0 & $\mathrm{pH}$ gradient \\
\hline trastuzumab & HER2 & $\begin{array}{l}\text { breast cancer } \\
\text { gastric cancer }\end{array}$ & hzlgG1k & $\begin{array}{l}\text { Roche, F. Hoffmann- } \\
\text { La Roche } \\
\text { LtD./Genetech Inc. }\end{array}$ & HERCEPTIN & 8.8 & $\mathrm{pH}$ gradient \\
\hline mAbPF1 & - & $\mathrm{n} / \mathrm{a}$ & hzlgG1k & Pierre Fabre & $\mathrm{n} / \mathrm{a}$ & 9.2 & $\mathrm{pH}$ gradient \\
\hline mAbPF2 & - & $n / a$ & hzlgG1k & Pierre Fabre & $n / a$ & 8.9 & $\mathrm{pH}$ gradient \\
\hline
\end{tabular}

